\title{
The 12th Advanced Summer School on Service-Oriented Computing
}

\author{
June 24-June 29 2018, Hersonissos, Crete, Greece
}

\author{
Bernhard Mitschang ${ }^{1}$
}

๑) Springer-Verlag GmbH Germany, part of Springer Nature 2019

The 12th Advanced Summer School on Service Oriented Computing (SummerSOC'18) continued a successful series of summer schools that started in 2007, regularly attracting world-class experts in Service Oriented Computing to present state-of-the-art research during a week-long program organized in several thematic tracks: patterns and IoT, Quantum Computing, formal methods for SOC, computing in the clouds, data science, e-Health and emerging topics. The advanced summer school is regularly attended by top researchers from academia and industry as well as by $\mathrm{PhD}$ and graduate students.

During the different sessions at SummerSOC renowned researchers gave invited tutorials on subjects from the themes mentioned above. The afternoon sessions were dedicated to the SummerSOC symposium and its original research contributions in the areas mentioned above. All accepted contributions were submitted in advance and have been peer-reviewed. In addition, the contributions had been extensively discussed during a poster session. The feedback of this discussion too has been folded into the version published in this special issue.

The first article suggests an approach to assess the cloud readiness of parallel applications based on the design decisions made; the first author received the "SummerSoC
Young Researcher Award" sponsored by ICSOC. The next article is devoted to the automatic detection of problems in restructured deployment models based on formalizing architecture and design patterns, followed by a contribution suggesting a semi-automated troubleshooting process to improve fault detection and resolution for serverless functions. The next article proposes a business process modeling extension that captures the particularities of blockchains. And the subsequent one, establishes a theory of solution algebras, which perceive concrete solutions of patterns and their aggregation operators as mathematical objects. The next contribution focusses on how data mining tasks can be made more efficient by stopping clustering algorithms as early as possible while still achieving an adequate quality of the detected clusters. Followed by a contribution using architectural modifiability tactics to examine evolution qualities of service- and microservice-based systems, the second last contribution focusses on mimicking FogDirector application management by presenting a simple operational semantics of the main features of FogDirector. Finally, the last article in this series introduces TOSCA intent models in order to support goal-modelling for Infrastructure-as-Code.
Bernhard Mitschang

Bernhard.Mitschang@ipvs.uni-stuttgart.de

1 Universität Stuttgart, Stuttgart, Germany 